\title{
Turán-type results for intersection graphs of boxes
}

\author{
István Tomon ${ }^{1, *}$ and Dmitriy Zakharov ${ }^{2,3}$ \\ ${ }^{1}$ ETH Zurich, Zurich, Switzerland ${ }^{2}$ MIPT Moscow, Russia and ${ }^{3}$ HSE Moscow, Russia \\ *Corresponding author. Email: istvan.tomon@math.ethz.ch
}

(Received 14 September 2020; revised 25 March 2021; accepted 27 April 2021; first published online 19 May 2021)

\begin{abstract}
In this short note, we prove the following analog of the Kövári-Sós-Turán theorem for intersection graphs of boxes. If $G$ is the intersection graph of $n$ axis-parallel boxes in $\mathbb{R}^{d}$ such that $G$ contains no copy of $K_{t, t}$, then $G$ has at most $\operatorname{ctn}(\log n)^{2 d+3}$ edges, where $c=c(d)>0$ only depends on $d$. Our proof is based on exploring connections between boxicity, separation dimension and poset dimension. Using this approach, we also show that a construction of Basit, Chernikov, Starchenko, Tao and Tran of $K_{2,2}$-free incidence graphs of points and rectangles in the plane can be used to disprove a conjecture of Alon, Basavaraju, Chandran, Mathew and Rajendraprasad. We show that there exist graphs of separation dimension 4 having superlinear number of edges.
\end{abstract}

2020 MSC Codes: Primary 05C10, 05C35

\section{Introduction}

The celebrated Kövári-Sós-Turán theorem [9] states that if $G$ is a graph on $n$ vertices containing no copy of $K_{t, t}$, then $G$ has $O\left(n^{2-1 / t}\right)$ edges. In the past few decades, a great amount of research was dedicated to showing that this bound can be significantly improved in certain restricted families of graphs, many of which are geometric in nature. See, for example, [6] for semi-algebraic graphs, [7] for graphs of bounded VC dimension and [5] for intersection graphs of connected sets in the plane. In particular, Fox and Pach [5] proved that if $G$ is the intersection graph of $n$ arcwise connected sets in the plane, and $G$ contains no $K_{t, t}$, then $G$ has at most $c n$ edges, where $c=c(t)>0$ depends only on $t$. In this paper, we are interested in the question that in what meaningful ways can this result be extended in higher dimensions. That is, for which families of geometric objects is it true that if their intersection graph $G$ is $K_{t, t}$-free, then $G$ has at most linear, or almost linear number of edges?

It turns out that already in dimension 3, one must put heavy restrictions on the family for this to hold. As a counterexample to many natural candidates, there exists a family of $n$ lines in $\mathbb{R}^{3}$, whose intersection graph is $K_{2,2}$-free and contains $\Omega\left(n^{4 / 3}\right)$ edges. To see this, consider a configuration of $n / 2$ points and $n / 2$ lines on the plane with $\Omega\left(n^{4 / 3}\right)$ incidences, which is the most possible number of incidences by the well known Szemerédi-Trotter theorem [11]. To get an intersection graph in $\mathbb{R}^{3}$, replace each point with a line parallel to the $z$-axis containing the point, and replace each line $l$ with a line $l^{\prime}$ such that the projection of $l^{\prime}$ to the $x y$-plane is $l$, and the lines $l^{\prime}$ are pairwise disjoint. This family of $n$ lines in $\mathbb{R}^{3}$ contains no $K_{2,2}$, and has $\Omega\left(n^{4 / 3}\right)$ intersections. 
One natural family of geometric objects for which the above question becomes interesting is the family of axis-parallel boxes. In this case, we are able to prove an almost linear upper bound on the number of edges.

Theorem 1. Let $d$, $t$ be positive integers, then there exists $c=c(d)>0$ such that the following holds. If $G$ is the intersection graph of $n d$-dimensional axis-parallel boxes such that $G$ contains no $K_{t, t}$, then $G$ has at most $\operatorname{ctn}(\log n)^{2 d+3}$ edges.

Our Theorem 1 is closely related to one of the main results in [3], which was done independently from us. In [3], they consider the number of edges of so called semilinear graphs of bounded complexity containing no copy of $K_{t, t}$. One of their main results (Theorem (B) (1)) is that if $G$ is an incidence graph of $n$ points and $n$ boxes in $\mathbb{R}^{d}$, and $G$ is $K_{t, t}$-free, then $|E(G)| \leq c_{d, t} n(\log n)^{2 d}$, where $c_{d, t}$ only depends on $d$ and $t$. Note that if $G$ is the incidence graph of the set of points $P$ and set of boxes $\mathcal{B}$ in $\mathbb{R}^{d}$, then $G$ is also the intersection graph of $(d+1)$-dimensional boxes. Indeed, replace each point $p \in P$ with a box $Q \times[0,1]$, where $Q \subset \mathbb{R}^{d}$ is a very small box containing $p$. Also, replace each box $B \in \mathcal{B}$ with a box $B \times\left[x, x+\frac{1}{2 n}\right]$, where $0 \leq x<1$, and the intervals $\left[x, x+\frac{1}{2 n}\right]$ are pairwise disjoint. The intersection graph of these $2 n$ boxes is isomorphic to $G$. Therefore, our Theorem 1 is slightly more general, but gives a slightly worse exponent of $\log n$ in exchange.

One might conjecture that the almost linear upper bound in Theorem 1 can be replaced with a linear one. This is true in case $d=2$ by the above-mentioned result of Fox and Pach [5]. However, much to our surprise, a construction presented in [3] implies that this is no longer true for $d \geq 3$.

Theorem 2 (Basit, Chernikov, Starchenko, Tao and Tran [3]). For every $n$, there exists a $K_{2,2}$-free incidence graph of $n$ points and $n$ rectangles in the plane with $\Omega\left(n \frac{\log n}{\log \log n}\right)$ edges.

Indeed, as we stated before, an incidence graph of points and rectangles is also an intersection graph of three-dimensional boxes. Therefore, we get the following immediate corollary of the previous theorem, which in turn disproves a conjecture of Kostochka [8].

Corollary 3. For every $n$, there exists a bipartite $K_{2,2}$-free intersection graph of $n$ boxes in $\mathbb{R}^{3}$ with $\Omega\left(n \frac{\log n}{\log \log n}\right)$ edges.

Let us remark that recently Davies [4] proved the following interesting extension of Corollary 3. If $g$ is a fixed positive integer, then there exists an intersection graph $G$ of $n$ boxes in $\mathbb{R}^{3}$ with superlinear number of edges such that $G$ contains no cycle of length less than $g$. Tomon [12] further improved this by showing that there exists an incidence graph $G$ of $n$ points and $n$ rectangles such that $G$ contains no cycle of length less than $g$, and $G$ has more than $c_{g} n \log \log n$ edges, where $c_{g}>0$ depends only on $g$.

We also show that Theorem 2 implies the existence of a counterexample to a conjecture of Alon, Basavaraju, Chandran, Mathew and Rajendraprasad [2]. The separation dimension of a graph $G$ is the smallest $d$ for which there exists an embedding $\phi: V(G) \rightarrow \mathbb{R}^{d}$ such that if $\{x, y\}$ and $\left\{x^{\prime}, y^{\prime}\right\}$ are disjoint edges of $G$, then the axis-parallel box spanned by $\phi(x)$ and $\phi(y)$ is disjoint from the axis-parallel box spanned by $\phi\left(x^{\prime}\right)$ and $\phi\left(y^{\prime}\right)$. In [2], it was conjectured that for every $d$, there exists a constant $c>0$ such that if $G$ is a graph on $n$ vertices with separation dimension $d$, then $G$ has at most $c n$ edges. They proved this in the case $d=2$. Also, Scott and Wood [10] confirmed the conjecture for $d=3$, and proved the bound $O\left(n(\log n)^{d-3}\right.$ ) for $d>3$ (Corollary 16). However, we show that the conjecture no longer holds for $d \geq 4$.

Theorem 4. For every $n$, there exists a graph $G$ on $n$ vertices with $\Omega\left(n \frac{\log n}{\log \log n}\right)$ edges such that the separation dimension of $G$ is at most 4.

We show that the connection between separation dimension and $K_{2,2}$-free intersection graphs of boxes, combined with the aforementioned result of Scott and Wood for $d=4$ also implies 
the following almost matching bound to Theorem 2, which improves Theorem (A) (1) in [3] for $K_{2,2}$-free graphs.

Corollary 5. If $G$ is the incidence graph of $n$ points and $n$ rectangles in the plane, and $G$ is $K_{2,2}$-free, then $G$ has at most $O(n \log n)$ edges.

\section{Boxicity, poset dimension and separation dimension}

In order to prove Theorems 1 and 4, let us introduce some notation. The boxicity of a graph $G$, denoted by box $(G)$ is the smallest $d$ such that $G$ can be realised as the intersection graph of $d$-dimensional boxes. Given two points $x$ and $y$ in $\mathbb{R}^{d}$, let $b(x, y)$ denote the open box spanned by $x$ and $y$.

Let $\prec$ denote the partial ordering on $\mathbb{R}^{d}$ defined as $x \prec y$ if $x_{i}<y_{i}$ for $i=1, \ldots, d$. (Here, $v_{i}$ is the $i$ th coordinate of $v$.) Given a partially ordered set $P$, the dimension (Duschnik-Miller dimension) of $P$, denoted by $\operatorname{dim}(P)$ is the smallest $d$ such that there exists an embedding $\phi: P \rightarrow \mathbb{R}^{d}$ satisfying that $x<_{P} y$ if and only if $\phi(x) \prec \phi(y)$. The comparability graph of the poset $P$ is the graph on the elements of $P$ in which the vertices $x, y \in P$ are connected by an edge if $x<P y$ or $y<_{P} x$. If $G$ is a bipartite graph with bipartition $(A, B)$, one can define the poset $P$ on $V(G)$ in which $a<p$ for $a, b \in V(G)$ if $a \in A, b \in B$ and $\{a, b\} \in E(G)$. Say that $P$ is a poset associated to $G$ (note that this poset depends on the bipartition, so it might not be unique).

The following relation between boxicity and poset dimension was discovered by Adiga, Bhowmick and Chandran [1]. Given a graph $G$, define the bipartite poset $(P(G), \prec)$ as follows: let the elements of $P(G)$ be $V(G) \times\{0,1\}$, and let $(u, 0) \prec(v, 1)$ if $u=v$ or $u v \in E(G)$.

Theorem 6. [1] $\frac{1}{2} \operatorname{box}(G) \leq \operatorname{dim}(P(G)) \leq 2 \operatorname{box}(G)+4$. Also, if $G$ is bipartite, and $P$ is a poset associated to $G$, then $\operatorname{dim}(P) \leq 2 \operatorname{box}(G)$.

The poset $P(G)$ not only estimates the boxicity of $G$ well, its comparability graph also (almost) retains the property of being $K_{t, t}$-free.

Claim 7. If $G$ is $K_{t, t}$-free, then the comparability graph of $P(G)$ has a $K_{t, t}$-free induced subgraph with at least $e(G) / 2$ edges.

Proof. Let $(A, B)$ be a partition of $V(G)$ such that at least half of the edges of $G$ have one endpoint in $A$ and one endpoint in $B$. Then the subgraph of the comparability graph of $P(G)$ induced on $\{(a, 0): a \in A\} \cup\{(b, 1): b \in B\}$ is $K_{t, t}$-free. Indeed, a copy of $K_{t, t}$ in this subgraph would correspond to a copy of $K_{t, t}$ in $G$ in which one of the vertex classes is in $A$, and the other is in $B$.

Claim 8. Let $V$ be a set of points in $\mathbb{R}^{d}$ and let $P=(V, \prec)$. If the comparability graph $G$ of $P$ does not contain $K_{t, t}$, then every matching $\left\{x^{1}, y^{1}\right\}, \ldots,\left\{x^{t}, y^{t}\right\}$ of size $t$ in $G$ satisfies $\bigcap_{i=1}^{t} b\left(x^{i}, y^{i}\right)=\emptyset$.

Proof. Let us assume that there exists a matching $\left\{x^{1}, y^{1}\right\}, \ldots,\left\{x^{t}, y^{t}\right\}$ such that $\bigcap_{i=1}^{t} b\left(x^{i}, y^{i}\right) \neq$ $\emptyset$, and let $z \in \bigcap_{i=1}^{t} b\left(x^{i}, y^{i}\right)$. Without loss of generality, assume that $x^{i} \prec y^{i}$ for $i=1, \ldots, t$, then $x^{i} \prec z \prec y^{i}$. But then $x^{i} \prec z \prec y^{j}$ for all $1 \leq i, j \leq t$, which means that $x^{1}, \ldots, x^{t}$ and $y^{1}, \ldots, y^{t}$ span a copy of $K_{t, t}$ in $G$.

Note that this claim also tells us that if the comparability graph of the poset $P$ is $K_{2,2}$-free and $\operatorname{dim}(P) \leq d$, then its separation dimension is at most $d$, as $V$ is a suitable embedding of the vertices. Let us use this to prove Theorem 4 . First, we show a somewhat weaker result. 
Theorem 9. For every $n$, there exists a graph $G$ on $n$ vertices with $\Omega\left(n \frac{\log n}{\log \log n}\right)$ edges such that the separation dimension of $G$ is at most 6 .

Proof. Let $G$ be the bipartite intersection graph of $n$ boxes in $\mathbb{R}^{3}$ such that $G$ contains no copy of $K_{2,2}$, and $|E(G)|=\Omega\left(n \frac{\log n}{\log \log n}\right)$. Such a graph exists by Corollary 3 . But then $\operatorname{dim}(P) \leq 2 \operatorname{box}(G)=6$ by Theorem 6, where $P$ is a poset associated to $G$. We are done as $G$ has separation dimension at most 6 as well.

In order to improve the dimension from 6 to 4, we just note that if $G$ is the incidence graph given by Theorem 2 instead of the intersection graph of Corollary 3 , then $P$ has dimension at most 4. The proof of this follows from a similar argument as the one in [1], but for the reader's convenience, we present a short proof here as well.

Claim 10. Let $G$ be the incidence graph of a set $\mathcal{P}$ of points and a set $\mathcal{R}$ of rectangles in the plane, and suppose that $G$ is $K_{2,2}$-free. Let $P$ be the associated poset of $G$ given by the bipartition $(\mathcal{P}, \mathcal{R})$, then $\operatorname{dim}(P) \leq 4$.

Proof. Without loss of generality, we may assume that no two points of $\mathcal{P}$ lie on the same axisparallel line.

We show that if $G$ is $K_{2,2}$-free, then we can assume that no rectangle contains the other. Indeed, suppose that $R \subset Q$ for some rectangles $R, Q \in \mathcal{R}$. Then $R$ contains at most one point of $\mathcal{P}$ as $G$ is $K_{2,2}$-free. But then we can replace $R$ with a rectangle $R^{\prime}$ such that $R^{\prime}$ is very thin and long, $R^{\prime} \cap \mathcal{P}=R \cap \mathcal{P}$, and $R^{\prime}$ has no containment relation with any other element of $\mathcal{R}$. The incidence graph of this configuration is also $G$.

Consider the map $\phi: \mathbb{R}^{2} \rightarrow \mathbb{R}^{4}$ defined by $(x, y) \mapsto(x,-x, y,-y)$. Also, given a rectangle $S=$ $\{(x, y) \mid a \leq x \leq b, c \leq y \leq d\}$ on the plane, let $\rho(S)=(a,-b, c,-d) \in \mathbb{R}^{4}$. Note that a point $p$ lies in the interior of a rectangle $S$ if and only if $\rho(S) \prec \phi(p)$. Clearly, if $p, q \in \mathcal{P}$ are distinct, then $\phi(p)$ and $\phi(q)$ are incomparable. Also, as there is no containment relation between any two rectangles in $\mathcal{R}$, if $R, Q \in \mathcal{R}$, then $\rho(R)$ and $\rho(Q)$ are also incomparable.

This finishes the proof of Theorem 4. Let us continue with the proof of Theorem 1, which we prepare with the following lemma. This lemma is a folklore known in many different forms, but we include its proof for completeness.

Lemma 11. Let $t$ be a positive integer. Let $V \subset \mathbb{R}$ such that $|V|=n$, and let $G$ be a graph on the vertex set $V$. If $G$ has more than 2 tn edges, then $G$ contains a matching $\left\{x^{1}, y^{1}\right\}, \ldots,\left\{x^{t}, y^{t}\right\}$ of size $t$ such that $b\left(x^{t}, y^{t}\right) \subset \cdots \subset b\left(x^{1}, y^{1}\right)$.

Proof. A zigzag path of size $\ell$ in $G$ is a path $v_{1}, \ldots, v_{\ell}$ such that $v_{1}<v_{3}<\cdots<v_{2\lfloor(\ell-1) / 2\rfloor+1}<$ $v_{2\lfloor\ell / 2\rfloor}<\cdots<v_{4}<v_{2}$. We show that if $G$ has at least $(\ell-2) n+1$ edges, then $G$ contains a zigzag path of length $\ell$. We prove this by induction on $\ell$. If $\ell \leq 2$, the statement is trivial, so assume that $\ell>2$.

First, consider the case when $\ell$ is odd. For each vertex $v \in V(G)$, if $v$ has a neighbour $w \in V(G)$ such that $w<v$, then let $u$ be the largest such neighbour of $v$, and remove the edge $\{u, v\}$ from $G$. Let the resulting graph be $G^{\prime}$. We removed at most $n$ edges, so $\left|E\left(G^{\prime}\right)\right| \geq(\ell-3) n+1$. But then $G^{\prime}$ contains a zigzag path $v_{1}, \ldots, v_{\ell-1}$. Note that there must be a vertex $v_{\ell} \in E(G)$ such that $v_{\ell-2}<v_{\ell}<v_{\ell-1}$ and $\left\{v_{\ell}, v_{\ell-1}\right\} \in E(G)$, otherwise we would have removed $\left\{v_{\ell-2}, v_{\ell-1}\right\}$ from $G$. But then $v_{1}, \ldots, v_{\ell}$ is a zigzag path in $G$.

If $\ell$ is even, we proceed similarly. For each vertex $v \in V(G)$, if $v$ has a neighbour $w \in V(G)$ such that $v<w$, then let $u$ be the smallest such neighbour of $v$, and remove the edge $\{v, u\}$ from $G$. Let the resulting graph be $G^{\prime}$. We removed at most $n$ edges, so $\left|E\left(G^{\prime}\right)\right| \geq(\ell-3) n+1$. But 
then $G^{\prime}$ contains a zigzag path $v_{1}, \ldots, v_{\ell-1}$. Note that there must be a vertex $v_{\ell} \in E(G)$ such that $v_{\ell-1}<v_{\ell}<v_{\ell-2}$ and $\left\{v_{\ell-1}, v_{\ell}\right\} \in E(G)$, otherwise we would have removed $\left\{v_{\ell-1}, v_{\ell-2}\right\}$ from $G$. But then $v_{1}, \ldots, v_{\ell}$ is a zigzag path in $G$.

We finish the proof by noting that if $G$ has at least $2 t n$ edges, then $G$ contains a zigzag path $v_{1}, \ldots, v_{2 t}$ of size $2 t$. But then setting $x^{i}=v_{2 i-1}$ and $y^{i}=v_{2 i}$ for $i=1, \ldots, t$, we have $b\left(x^{t}, y^{t}\right) \subset$ $\cdots \subset b\left(x^{1}, y^{1}\right)$.

Theorem 12. Let $d$, $t$ be positive integers, then there exists $c=c(d)$ such that the following holds. Let $V$ be a set of $n$ points in $\mathbb{R}^{d}$ and let $G$ be a graph on the vertex set $V$ such that if $\left\{x^{1}, y^{1}\right\}, \ldots,\left\{x^{t}, y^{t}\right\}$ is a matching of size $t$ in $G$, then $\bigcap_{i=1}^{t} b\left(x^{i}, y^{i}\right)=\emptyset$. Then $e(G) \leq \operatorname{ctn}(\log n)^{d-1}$.

Proof. The statement follows from a standard divide and conquer argument.

Let us proceed by induction on $d$. First, consider the base case $d=1$, we show that $c=2 t$ suffices. Indeed, by Lemma 11 , if $G$ has more than $2 t n$ edges, then $G$ contains a matching $\left\{x^{1}, y^{1}\right\}, \ldots,\left\{x^{t}, y^{t}\right\}$ of size $t$ such that $b\left(x^{t}, y^{t}\right) \subset \cdots \subset b\left(x^{1}, y^{1}\right)$, which then implies $\bigcap_{i=1}^{t} b\left(x^{i}, y^{i}\right) \neq \emptyset$, contradiction.

Now suppose that $d \geq 2$. Let $f_{d}(n)$ denote the minimum $m$ such that any graph $G$ with the desired properties has at most $m$ edges. We show that $f_{d}(n) \leq c_{d} \operatorname{tn}(\log n)^{d-1}$, where $c_{d}>0$ depends only on $d$. Let $G$ be a graph with the desired properties. Without loss of generality, we can assume that no two points in $V$ are on the same axis-parallel hyperplane. Let $H$ be a $(d-1)$-dimensional hyperplane perpendicular to the last coordinate axis such that at most half of the points of $V$ are on each side of $H$. Let $A$ and $B$ be the set of points of $V$ on the two sides of $H$. Let $p(x)$ denote the projection of $x$ onto $H$, and let $G^{\prime}$ be the graph on vertex set $p(V)$ in which $p(x)$ and $p(y)$ are joined by an edge if $x y \in E(G)$ and $x \in A$ and $y \in B$. If $x, x^{\prime} \in A$ and $y, y^{\prime} \in B$, then $b(x, y) \cap b\left(x^{\prime}, y^{\prime}\right) \neq \varnothing$ if and only if $b(p(x), p(y)) \cap b\left(p\left(x^{\prime}\right), p\left(y^{\prime}\right)\right) \neq \emptyset$. Therefore, for every matching in $G^{\prime}$ of size $t$, the boxes spanned by the edges have an empty intersection. Hence, we deduce that

$$
e(G)=e(G[A])+e(G[B])+e(G[A, B]) \leq 2 f_{d}(\lceil n / 2\rceil)+e\left(G^{\prime}\right) \leq 2 f_{d}(\lceil n / 2\rceil)+f_{d-1}(n) .
$$

From this, we get that $f_{d}(n)=O\left(f_{d-1}(n) \log n\right)=O\left(\operatorname{tn}(\log n)^{d-1}\right)$, where the last equality holds by our induction hypothesis, and the constant hidden in the $O($.) notation only depends on $d$.

In case $t=2$, Theorem 12 can be improved. In this case, the graph $G$ has separation dimension at most $d$, which implies that $e(G) \leq c n(\log n)^{d-3}$ by the result of Scott and Wood [10] mentioned in the Introduction.

After these preparations, everything is set to prove our main theorem.

Proof of Theorem 1. Let $G$ be the intersection graph of $n$ boxes in $\mathbb{R}^{d}$, and suppose that $G$ is $K_{t, t}$-free. Then by Theorem 6 and Claim 7, there exists a poset $P$, whose comparability graph is $K_{t, t}$-free, has at least $e(G) / 2$ edges, and $\operatorname{dim}(P) \leq 2 \operatorname{box}(G)+4 \leq 2 d+4$. But then by Claim 8 and Theorem 12, the comparability graph of $P$ has at most $\operatorname{ctn}(\log n)^{2 d+3}$ edges, where $c=c(d)>0$ only depends on $d$. This implies $e(G)<2 \operatorname{ctn}(\log n)^{2 d+3}$.

Finally, let us prove Corollary 5.

Proof of Corollary 5. Let $G$ be the incidence graph of the set $\mathcal{P}$ of $n$ points and set $\mathcal{R}$ of $n$ rectangles in the plane, and suppose that $G$ is $K_{2,2}$-free. Then by Claim 10, the poset $P$ associated to $G$ with respect to the bipartition $(\mathcal{P}, \mathcal{R})$ has dimension at most 4 . But as $G$ is $K_{2,2}$-free, $G$ has separation dimension at most 4 , so by the result of Scott and Wood [10], $G$ has at most $O(n \log n)$ edges. 


\section{Acknowledgements}

The authors would like to thank Andrey Kupavskii, János Pach and Alexandr Polyanskii for valuable discussions, and the anonymous referees for their useful comments and suggestions. István Tomon was supported by the SNSF grant 200021_196965, and acknowledges the support of MIPT Moscow. Also, both authors acknowledge the support of the grant of the Russian Government $\mathrm{N}$ 075-15-2019-1926.

\section{References}

[1] Adiga, A., Bhowmick, D. and Chandran, L. S. (2011) Boxicity and poset dimension. SIAM J. Discrete Math. 25 1687-1698.

[2] Alon, N., Basavaraju, M., Chandran, L. S., Mathew, R. and Rajendraprasad, D. (2018) Separation dimension and sparsity. J. Graph Theory 89 14-25.

[3] Basit, A., Chernikov, A., Starchenko, S., Tao, T. and Tran, C.-M. (2020) Zarankiewicz's problem for semilinear hypergraphs. arXiv preprint, arXiv:2009.02922.

[4] Davies, J. (2020) Box and segment intersection graphs with large girth and chromatic number. arXiv preprint, arXiv:2011.14174.

[5] Fox, J. and Pach, J. (2008) Separator theorems and Turán-type results for planar intersection graph. Adv. Math. 219 1070-1080.

[6] Fox, J., Pach, J., Sheffer, A., Suk, A. and Zahl, J. (2017) A semi-algebraic version of Zarankiewicz's problem. J. Eur. Math. Soc. 19 1785-1810.

[7] Janzer, O. and Pohoata, C. (2020) On the Zarankiewicz problem for graphs with bounded VC-dimension. arXiv preprint, arXiv:2009.00130.

[8] Kostochka, A. V. (2004) Coloring intersection graphs of geometric figures with a given clique number. Contemp. Math. 342 127-138.

[9] Kővári, T., Sós, V. and Turán, P. (1954) On a problem of K. Zarankiewicz. Colloq. Math. 3 50-57.

[10] Scott, A. and Wood, D. R. (2018) Separation dimension and degree. Math. Proc. Camb. Phil. Soc. 1-10.

[11] Szemerédi, E. and Trotter, W. T. (1983) Extremal problems in discrete geometry. Combinatorica 3 381-392.

[12] Tomon, I. (2020) Ramsey properties of semilinear graphs. arXiv preprint, arXiv:2102.12464.

Cite this article: Tomon I and Zakharov D (2021). Turán-type results for intersection graphs of boxes. Combinatorics, Probability and Computing 30, 982-987. https://doi.org/10.1017/S0963548321000171 